\title{
Natural variation at $q H d 1$ affects heading date acceleration at high temperatures with pleiotropism for yield traits in rice
}

\author{
Jun-Yu Chen®D, Hong-Wei Zhang, Hua-Li Zhang, Jie-Zheng Ying, Liang-Yong Ma* and Jie-Yun Zhuang*
}

\begin{abstract}
Background: Rice is highly sensitive to temperature fluctuations. Recently, the frequent occurrence of high temperature stress has heavily influenced rice production. Proper heading date in specific environmental conditions could ensure high grain yield. Rice heading greatly depends on the accurate measurement of environmental changes, particularly in day length and temperature. In contrary to the detailed understanding of the photoperiod pathway, little has been known about how temperature regulates the genetic control of rice heading.

Results: Near isogenic lines that were segregated for $9 \mathrm{Hd}$ 1, were developed from a cross between indica rice varieties Zhenshan 97 (ZS97) and Milyang 46 (MY46). Using a five sowing-date experiment in the paddy field, we observed the involvement of $q \mathrm{Hd} 1$ in temperature responses. With the gradual increase of temperature from Trial I to $\mathrm{V}$, heading date of MY46 homozygotes continued to decrease for about $5 \mathrm{~d}$ per trial from 76 to $58 \mathrm{~d}$, while that of ZS97 homozygotes was promoted at the same rate from Trial I to III and then stabilized at $69 \mathrm{~d}$. This thermal response was confirmed in a temperature-gradient experiment conducted in the phytotron. It is also found that tolerance of the ZS97 allele to heading acceleration at high temperature was associated with higher grain weight that resulted in higher grain yield. Then, by qRT-PCR and RNA-seq, we found the pathway OSMADS51-Ehd1-RFT1/ Hd3a underlying the qHd1-mediated floral response to temperature. By sequence comparison, OSMADS51 for qHd1 displayed a 9.5-kb insertion in the 1st intron of the ZS97 allele compared to the MY46 allele. Furthermore, this large insertion is commonly found in major early-season indica rice varieties, but not in the middle- and late-season ones, which corresponds to the requirement for high-temperature tolerance during the heading and grain-filling stages of early-season rice.
\end{abstract}

Conclusions: Beneficial alleles at $q \mathrm{Hd} 1$ confer tolerance to high temperatures at the heading and grain-filling stages, playing a significant role in the eco-geographical adaptation of early-season indica rice during modern breeding. These results, together with the underlying OsMADS51-Ehd1-RFT1/Hd3a floral pathway, provide valuable information for better understanding the molecular mechanism of temperature responsive regulation of heading date and yield traits in rice.

Keywords: Rice, Temperature response, Heading date, Grain filling, Adaption, Quantitative trait locus

\footnotetext{
*Correspondence: maliangyong@caas.cn; zhuangjieyun@caas.cn

State Key Laboratory of Rice Biology and Chinese National Center for Rice

Improvement, China National Rice Research Institute, Hangzhou 310006,

China
}

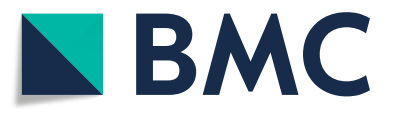

(c) The Author(s). 2018 Open Access This article is distributed under the terms of the Creative Commons Attribution 4.0 International License (http://creativecommons.org/licenses/by/4.0/), which permits unrestricted use, distribution, and reproduction in any medium, provided you give appropriate credit to the original author(s) and the source, provide a link to the Creative Commons license, and indicate if changes were made. The Creative Commons Public Domain Dedication waiver (http://creativecommons.org/publicdomain/zero/1.0/) applies to the data made available in this article, unless otherwise stated. 


\section{Background}

In recent decades, the influence of climate changes on rice productivity has become increasingly concerned, such as more frequent high temperature stress accompanied with global warming [1]. A small increase in the daily mean temperature of near $2{ }^{\circ} \mathrm{C}$ would make a dramatic decrease in grain yield [2]; likewise, yield reduction could occur by $1{ }^{\circ} \mathrm{C}$ increase in the mean minimum temperature [3]. In rice production and breeding practice, heading date and yield traits are generally considered as key indicators for the response to temperature variation; for instance, early heading and yield loss have been commonly observed at high temperatures. Rice growth process consists of three sequential stages, i.e., vegetative, reproductive, and grain filling [4]. Flower initiation is the indication of transition from vegetative to reproductive stage, which largely determines the heading date variation among different rice genotypes and is governed by thermal sensitivity besides the basic vegetative growth and photoperiod sensitivity [5-7]. Poor seed setting and insufficient grain filling that result in yield loss are typical symptoms at high temperature during the second half of rice growth [8-10]. That is to say, rice is highly sensitive to temperature changes, particularly at the heading and grain-filling stages. To ensure proper seasonal and regional adaptation of rice varieties, heading date has always been a primary target in rice breeding. Clearly, understanding how temperature plays a role in regulating rice heading would be essential to mitigate the impacts of climate changes, particularly when temperature appears to be rising.

Unfortunately, neither gene cloning nor quantitative trait locus (QTL) primary mapping in rice has explored the genetic contributions of the thermo-sensitivity to heading date. Gene cloning exclusively focused on the photoperiodic regulation of heading and identified two independent gene pathways $[11,12]$. One is the Heading date 1 (Hd1) -Heading date $3 a(H d 3 a)$ pathway that is evolutionarily correlated with the Arabidopsis CONSTANS (CO) -FLOWERING LOCUS T (FT) pathway. The other is the Early heading date 1 (Ehd1) $-H d 3 a /$ Rice flowering locus T1 (RFT1) pathway that is unique to rice. QTL primary mapping dealt with various aspects associated with heading date, including basic vegetative phase, photoperiod sensitivity, vegetative growth time, reproductive growth time, and days to maturity (http://archive.gramene.org/qtl/), but thermal sensitivity has not been taken into account. Therefore, it is urgent to identify genetic factors determining the response of rice heading to temperature variations. In Arabidopsis thaliana, studies have revealed the existence of at least some interactions between photoperiod and temperature pathway. For instance, PHYTOCHROME INTERACTING FACTOR 4 could not display the thermal induction of flowering under continuous light, but this function was observed in short-day conditions [13]; a mutation at
EARLY FLOWERING 3 greatly influenced the sensitivity to temperature under abnormal photoperiod pathway [14]. Other genes associated with the response to both photoperiod and temperature changes included photoreceptors such as CRYPTOCHROME 1 [15], CRYPTOCHROME 2 [16] and PHYTOCHROME B $[17,18]$. These findings provide evidences for the importance of thermo-sensitivity in regulating flowering in plants and offer reference to identify relevant molecular contributors in rice.

Using near isogenic lines (NILs) and NIL-F 2 populations derived from a cross between indica rice varieties Zhenshan 97 (ZS97) and Milyang 46 (MY46), we previously fine-mapped $q H d 1$, a minor-effect QTL for heading date independent of day-length [19]. Surprisingly, the effect of $q H d 1$ was greatly decreased in a following-up test in the winter rice-growing season in Lingshui, Hainan, China. Heading date difference between NILs $q H d 1^{\mathrm{ZS} 97}$ and $q H d 1^{\text {MY46 }}$ was only 2.6 d (Additional file 1 : Figure S1), much smaller than the difference of $5.3 \mathrm{~d}$ observed previously [19]. Therefore, we carried out the present study to investigate the relationship between the function of $q \mathrm{Hd} 1$ and temperature variation. We firstly conducted a multiple sowing-date experiment in the paddy field to test $q H d 1$, followed by a validation in the controlled environment. Results suggest that $q H d 1$ regulates rice heading in response to temperature, and contributes to high-temperature tolerance at the heading and grain-filling stages. Then, by gene sequencing and expression analysis including RNA-seq, the temperature sensing pathway of OsMADS51-Ehd1-Hd3a/RFT1 was identified, with gene OsMADS51 underlying QTL $q H d 1$. In addition, by genotyping 109 major indica rice varieties released in China since 1950s, evidence was shown for the significance of $q H d 1$ in the adaptation of early-season indica rice to high temperature during rice heading and grain filling. This information would be greatly useful for exploring the molecular mechanism of temperature-related regulation of rice heading in breeding practice.

\section{Methods}

\section{Rice materials}

Two sets of NILs and 109 indica rice varieties were used. The two NIL sets were segregated for $q H d 1$, a minor-effect QTL for heading date [19]. They were derived from a $\mathrm{BC}_{2} \mathrm{~F}_{8}$ plant of the indica rice cross ZS97///ZS97//ZS97/MY46 and named CJ1 and CJ2 (Additional file 2: Figure S2). Each NIL set contained 50 homozygous ZS97 lines and 50 homozygous MY46 lines. The 109 indica rice varieties consisted of three collections (Additional file 3: Table S1). The first collection contained 13 early-season varieties that had large planting areas for a long period before 1985 . The second collection included 48 early-season, 24 middle-season and 10 late-season varieties that were planted in large areas 
for at least one year during 1990-2014 [20, 21]. The third collection had 14 early-season varieties, including Jiayu 253, Zhongxuan 181, and 12 descendants of either Jiayu 253 or Zhongxuan 181, which were released after 2000.

\section{Field experiments and trait evaluation}

The two NIL sets were tested in the paddy field at the China National Rice Research Institute in Hangzhou, China. They were grown from April to October in 2015 with five sowing dates. The sowing and transplanting dates were 28 April and 25 May for Trial I, 8 May and 2 June for Trial II, 20 May and 11 June for Trial III, 15 June and 6 July for Trial IV, and 9 July and 29 July for Trial V, respectively (Table 1). A randomized complete block design with two replications was used. In each replication, each line was grown in a single row of 12 plants, with spacing of $16.7 \mathrm{~cm}$ between plants and $26.7 \mathrm{~cm}$ between rows. Field management followed the normal agricultural practice. The soil type belongs to purplish clayey soil and irrigation was applied to maintain a proper-watered condition from sowing to harvesting. During the period of floral transition in the rice materials tested, day length in Hangzhou was longer than $13 \mathrm{~h}$ (www.timeanddate.com), corresponding to natural long day (NLD) conditions [22-24]. From the weather station located near the experimental field, the data of daily mean temperature were also collected.

Heading date (HD) was recorded for all the five trials, and yield traits were measured for Trial I, III and IV. HD was recorded for each plant when a panicle emerged. The data were averaged over all plants of each line in each replication. At maturity, five middle plants of each line were harvested in bulk and sun-dried. Five yield traits were measured, including number of panicles per plant (NP), number of grains per panicle (NGP), 1000-grain weight (TGW, g), spikelet fertility rate (SF, $\%$ ), and grain yield per plant (GY, g). For measuring TGW, fully filled grains maintaining a constant condition among different samples were used as described previously [25].

Table 1 The multiple sowing-date experiment conducted in Hangzhou in 2015

\begin{tabular}{llll}
\hline Trial & Sowing date & Transplanting date & Traits measured \\
\hline I & 28 April & 25 May & $\mathrm{HD}$, yield traits \\
II & 8 May & 2 June & $\mathrm{HD}$ \\
III & 20 May & 11 June & $\mathrm{HD}$, yield traits \\
IV & 15 June & 6 July & $\mathrm{HD}$, yield traits \\
V & 9 July & 29 July & $\mathrm{HD}$ \\
\hline
\end{tabular}

$H D$ heading date; yield traits measured included number of panicle per plant (NP), number of grains per panicle (NGP), 1000-grain weight (TGW, g), spikelet fertility (SF, \%) and grain yield per plant (GY, g)

\section{Phytotron experiment with temperature-gradient treatment}

To validate the responsiveness of $q H d 1$ to temperature variation, NILs derived from CJ1 and consisted of two homozygous genotypes were grown in phytotrons at humidity of $70 \%$ with three different temperature treatments under short day (SD) conditions. The temperature-gradient test consisted of daily cycles of $11 \mathrm{~h}$ light at 26,28 and $30^{\circ} \mathrm{C}$ and $13 \mathrm{~h}$ dark at 22,24 and $26^{\circ} \mathrm{C}$, respectively. Two lines were planted for each genotype, with eight plants per line. Heading date was scored for each plant.

\section{Quantitative real-time PCR (qRT-PCR) analysis}

Penultimate leaves of the two homozygous genotypes of CJ1 were collected at 8:30-9:00 am in 30, 35 and 45 days after sowing with the same sowing date to Trial IV, well covering the transition period from vegetative to reproductive phase. One sample consisted of three leaves and three samples were analyzed for each genotype. According to the manufacturer's instruction, we used the AxyPrep $^{\text {Tm }}$ Multisource Total RNA Miniprep Kit (Axygen) to extract total RNA, which was then retro-transcribed by using PrimeScript ${ }^{\text {tw }}$ RT Reagent Kit with gDNA Eraser (Takara). Quality and concentration of the RNA extracted was checked with electrophoresis on $1 \%$ agarose gel and measured using the Nanodrop ND-2000 spectrophotometer (NanoDrop Technologies, USA). Concentration of the RNA samples used for cDNA synthesis was normalized by dilution with RNase-free ultra pure water.

qRT-PCR assays of $20 \mu \mathrm{L}$ reaction volumes, which contained $0.5 \mu \mathrm{L}$ of synthesized cDNA, $0.4 \mu \mathrm{M}$ of gene-specific primers and $10 \mu \mathrm{L}$ of SYBR Premix Ex $\mathrm{Taq}^{\mathrm{Tm}}$ (TaKaRa), were conducted by using ABI 7500 Real-time PCR System (Applied Biosystems). Following the manufacturer's instruction, the qRT-PCR conditions were set up as: denaturing at $95{ }^{\circ} \mathrm{C}$ for $30 \mathrm{~s}$, then 40 cycles of $95{ }^{\circ} \mathrm{C}$ for $5 \mathrm{~s}, 55^{\circ} \mathrm{C}$ for $30 \mathrm{~s}$ and $72{ }^{\circ} \mathrm{C}$ for $30 \mathrm{~s}$. To standardize the quantification of gene expression, we used the rice Ubiquitin (UBQ) gene (Os03g0234200, http://rapdb.dna.affrc.go.jp/) as an internal control. The data were analyzed according to the relative quantification method [26]. Primers for qRT-PCR of $U B Q$, OsMADS51, Ehd1, Hd3a, RFT1, Hd1 and OsSPL2 were listed in Additional file 4: Table S2.

\section{RNA-Seq analysis}

Two homozygous genotypes derived from CJ1 were grown in controlled chambers treated with high and low temperature under long day (LD) conditions, which were set as $34{ }^{\circ} \mathrm{C}$, 14-h light $/ 28{ }^{\circ} \mathrm{C}$, 10 -h dark and $26{ }^{\circ} \mathrm{C}$, 14-h light $/ 20{ }^{\circ} \mathrm{C}, 10$-h dark, respectively. Penultimate leaves of the two genotypes were collected in 23 days after sowing (DAS) at high temperature and 44 DAS at low temperature with two biological replicates. In this 
condition, heading date of the early-heading genotype was $49 \mathrm{~d}$ at high temperature and $70 \mathrm{~d}$ at low temperature. Based on the understanding that the duration from panicle initiation to heading in early-season indica rice is constantly about $28 \mathrm{~d}$, the 23 DAS for high temperature and 44 DAS at low temperature both correspond to about $26 \mathrm{~d}$ before heading, covering the transition period from vegetative to reproductive phase. Total RNA was extracted using the TRIzol reagent (Invitrogen), according to the manufacturer's protocol. RNA quantification and qualification, library preparation for transcriptome sequencing, clustering and sequencing, data analysis and quality control were conducted by Biomarker (http://www.biomarker.com.cn/) according to their protocol.

Differential expression analysis between the two genotypic groups was performed using the DESeq $\mathrm{R}$ package (1.10.1) with a model based on the negative binomial distribution. The resulting $P$ values were adjusted for controlling the false discovery rate, using the Benjamini and Hochberg's approach. Genes with an adjusted $P$-value $<0.01$ and $\log 2$ fold change above or below 1.0 were designated as differentially expressed genes (DEGs). For validation of critical genes identified from the RNA-seq analysis, qRT-PCR was performed by using the same samples for the RNA-seq.

\section{Statistical analysis}

For the replicated field trials, phenotypic differences between the two different genotypic groups in each NIL set were evaluated by two-way analysis of variance (ANOVA), which was performed with SAS procedure GLM (SAS Institute Inc. 1999) as described previously [27]. When a significant difference $(P<0.05)$ was found between the two genotypes, the QTL effects, including additive effect and the proportion of phenotypic variance explained, were estimated with the same data and model.

For NIL set that was grown in the controlled chamber, one-way ANOVA was conducted to test the phenotypic differences between the two homozygous genotypic groups.

\section{Results}

\section{Effect of $q H d 1$ on the tolerance to high temperatures shown in the multiple sowing-date experiment Photoperiod and temperature conditions}

With the sowing date changing at a specific rice cropping area, the available photo-temperature resources and the development progress of a rice variety varied greatly. Five different sowing dates were applied in this study, lasting from early to late cropping season in 2015 in Hangzhou (Table 1).
Day length and temperature are two key factors of environmental conditions and closely associated with seasonal changes. As for day length, $13.0 \mathrm{~h}$ could be considered as the threshold distinguishing SD from LD conditions for rice [22-24]. In the multiple-sowing experiment, the day length increased from $13.3 \mathrm{~h}$ at 28 April to $14.1 \mathrm{~h}$ at 23 June, and then decreased to $13.0 \mathrm{~h}$ at 24 August (Additional file 5: Figure S3). Generally, the duration from panicle initiation to heading in rice stably takes about $28 \mathrm{~d}$ [5, 28-30]; that is to say, rice plants flowering before 20 September could experience the transition from vegetative to reproductive phase under LD conditions. Given that, we purposed that all the lines tested in this experiment have completed the phase transition under LD conditions. As for daily mean temperature, a warming trend from early to late cropping seasons was found, despite of obvious fluctuations (Additional file 5: Figure S3). The average temperatures from sowing to heading were $23.6{ }^{\circ} \mathrm{C}$ in Trial I, $24.1{ }^{\circ} \mathrm{C}$ in Trial II, $24.9{ }^{\circ} \mathrm{C}$ in Trial III, $27.1{ }^{\circ} \mathrm{C}$ in Trial IV, and $27.7^{\circ} \mathrm{C}$ in Trial V. From these observations, we inferred that temperature would be a more critical environmental variable than day length in this test.

\section{QTL effects on heading date}

In all the five trials, the ZS97 homozygous lines always had a late heading as compared to the MY46 homozygous lines. With the postponement of sowing date from Trial I to V, HD of MY46 homozygotes continued to decrease for about $5 \mathrm{~d}$ per trial from 76 to $58 \mathrm{~d}$, while that of ZS97 homozygotes was promoted at the same rate in the first three trials but then stabilized at $69 \mathrm{~d}$ (Fig. 1a).

Two-way ANOVA was conducted to test the heading-date difference between the two genotypic groups of each NIL population. As shown in Table 2, highly significant genotypic effects $(P<0.0001)$ were detected in both populations in each trial, explaining $54.0-90.4 \%$ of the phenotypic variances. Notably, the additive effects of $q H d 1$ were similar among the first three trials and then increased in the last two ones: the averaged effects of ZS97 allele on delaying heading over CJ1 and CJ2 were ranged from 1.4 to $1.8 \mathrm{~d}$ in Trial I, II and III, while the effects became 4.0 and $5.7 \mathrm{~d}$ in Trial IV and V, respectively. These results support the notion that $q H d 1$ mediated a floral response to temperature variation with the allele from ZS97 conferring a stable heading date at high temperatures.

\section{QTL effects on yield traits}

According to the HD distribution curves of ZS97 homozygotes across the five treatments (Fig. 1a), we easily identified the declining trend from Trial I to III and the steady trend from Trial III to $\mathrm{V}$ with the turning point in Trial III. Therefore, Trial I, III and IV were selected 


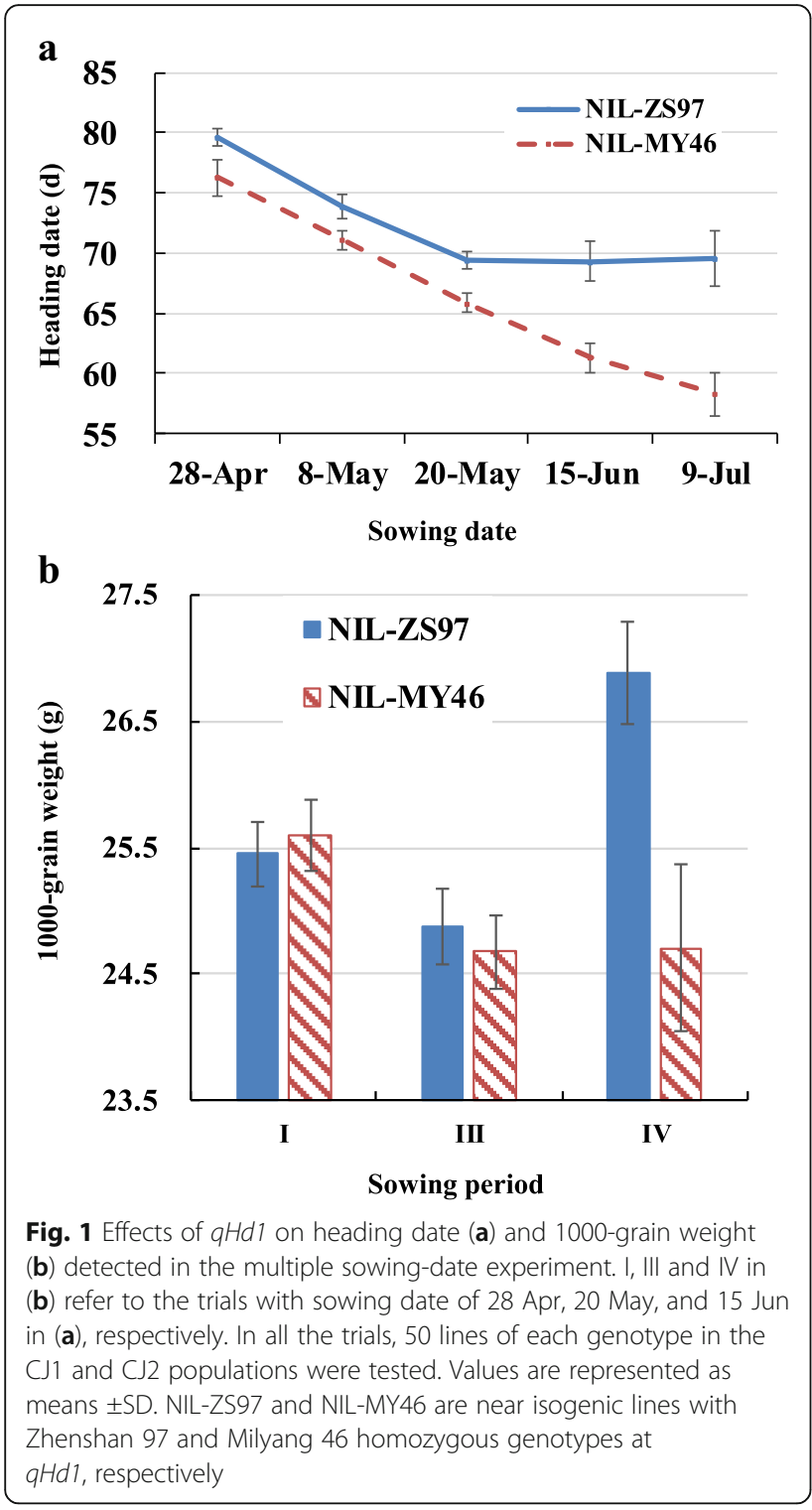

as the representatives to further analyze the effects of $q H d 1$ on yield traits.

Two-way ANOVA was performed to test phenotypic differences of yield traits between the two homozygous genotypic groups of each NIL population, and the results are presented in Table 3. When a significant effect $(P<0.05)$ was detected, the enhancing alleles were always derived from ZS97 except for the small effects detected on TGW in CJ1 in Trial I and on SF in CJ2 in Trial III. This is in accordance with the understanding that late-maturing allele is generally associated with improving performance of yield traits.

In Trial I, significant genotypic effects were detected on NP, TGW and GY, but none of them were consistent across the two NIL populations. In Trial III, yield
Table 2 Effects of qHd1 on heading date tested in the multiple sowing-date experiment

\begin{tabular}{|c|c|c|c|c|c|c|}
\hline \multirow{2}{*}{$\begin{array}{l}\text { Trial } \\
\text { (Sowing date) }\end{array}$} & \multirow{2}{*}{$\begin{array}{l}\text { Population } \\
\text { name }\end{array}$} & \multicolumn{2}{|c|}{ Phenotypic mean ${ }^{a}$} & \multirow[t]{2}{*}{$P$} & \multirow[t]{2}{*}{$A^{\mathrm{b}}$} & \multirow[t]{2}{*}{$R^{2}(\%)$} \\
\hline & & $\overline{\mathrm{NIL}}{ }^{\mathrm{ZS} 97}$ & $\overline{N I L}{ }^{\mathrm{MY} 46}$ & & & \\
\hline \multirow[t]{2}{*}{ Trial-I (28 Apr) } & CJ1 & 79.3 & 76.0 & $<0.0001$ & -1.6 & 58.6 \\
\hline & $\mathrm{CJ} 2$ & 79.9 & 76.5 & $<0.0001$ & -1.7 & 59.4 \\
\hline \multirow[t]{2}{*}{ Trial-II (8 May) } & CJ1 & 74.1 & 71.3 & $<0.0001$ & -1.4 & 54.0 \\
\hline & $\mathrm{CJ} 2$ & 73.7 & 70.9 & $<0.0001$ & -1.4 & 62.0 \\
\hline \multirow[t]{2}{*}{ Trial-III (20 May) } & CJ1 & 69.3 & 66.1 & $<0.0001$ & -1.6 & 76.8 \\
\hline & $\mathrm{CJ} 2$ & 69.5 & 65.7 & $<0.0001$ & -1.9 & 81.2 \\
\hline \multirow[t]{2}{*}{ Trial-IV (15 Jun) } & CJ1 & 69.1 & 61.5 & $<0.0001$ & -3.8 & 79.6 \\
\hline & $\mathrm{CJ} 2$ & 69.5 & 61.2 & $<0.0001$ & -4.2 & 90.4 \\
\hline \multirow[t]{2}{*}{ Trial-V (9 Jul) } & CJ1 & 69.6 & 58.3 & $<0.0001$ & -5.6 & 81.6 \\
\hline & $\mathrm{CJ} 2$ & 69.6 & 58.2 & $<0.0001$ & -5.7 & 88.4 \\
\hline
\end{tabular}

${ }^{\mathrm{a}} \mathrm{NIL}^{\mathrm{ZS97}}$ and $\mathrm{NIL}^{\mathrm{MY} 46}$ are near isogenic lines having homozygous $q \mathrm{Hd} 1$ alleles from Zhenshan 97 and Milyang 46, respectively

badditive effect of replacing a Zhenshan 97 allele with a Milyang 46 allele cproportion of phenotypic variance explained by the QTL effect

component traits NGP and TGW, but not NP, showed significant variation in both populations, while the significance of GY variation was just marginal $(P=0.0500$ in $C J 1$ and $P=0.0701$ in CJ2). In Trial IV, highly significant and relatively large variation was observed on TGW and GY in both populations, while a small variation with opposite allelic direction was found on SF. Notably, TGW of ZS97 homozygotes were much larger than that of MY46 ones, with the additive effects and $R^{2}$ averaged as $1.09 \mathrm{~g}$ and $74.0 \%$ over the two populations, respectively, which were the main sources of variation for GY.

Variation of TGW across the three trials was especially noteworthy when it was compared with variation of temperature and HD. As compared to MY46 homozygotes, ZS97 homozygotes greatly increased TGW in Trial IV but not in Trial I and III (Fig. 1b). The average increase over the two populations was $8.8 \%$ in Trial IV, whereas differences between the two genotypes were all less than $1 \%$ in Trial I and III (Table 3 ). When considering the increase of temperature with postponed sowing date (Additional file 5: Figure S3), it is reasonable to infer that the $q H d 1^{\mathrm{ZS} 97}$ allele is favorable for achieving high grain weight at high temperature. When further considering the stable HD of ZS97 homozygotes under high temperature, it is evident that the $q H d 1^{\mathrm{ZS} 97}$ allele would be advantageous for realizing a higher grain yield through its tolerance to heading date acceleration at high temperatures.

\section{Validation of the floral response of $q H d 1$ to temperature by the temperature-gradient test in phytotron}

NIL sets which included two homozygous genotypes derived from $\mathrm{CJ} 1$ differing at $q H d 1$ was examined in the controlled chambers with increasing ambient temperature 
Table 3 Effects of aHd1 on yield traits in Trail-I, III and IV of the multiple sowing-date experiment

\begin{tabular}{|c|c|c|c|c|c|c|c|c|c|c|c|}
\hline \multirow{2}{*}{$\begin{array}{l}\text { Trial } \\
\text { (Sowing date) }\end{array}$} & \multirow[t]{2}{*}{ Trait } & \multicolumn{2}{|c|}{ CJ1-Phenotypic mean ${ }^{a}$} & \multirow[t]{2}{*}{$P$} & \multirow[t]{2}{*}{$A^{\mathrm{b}}$} & \multirow{2}{*}{$\begin{array}{l}R^{2} \\
(\%)^{c}\end{array}$} & \multicolumn{2}{|c|}{ CJ2-Phenotypic mean } & \multirow[t]{2}{*}{$P$} & \multirow[t]{2}{*}{$A$} & \multirow{2}{*}{$\begin{array}{l}R^{2} \\
(\%)\end{array}$} \\
\hline & & $\mathrm{NIL}^{\mathrm{ZS} 97}$ & $\mathrm{NIL}^{\mathrm{MY} 46}$ & & & & $\mathrm{NIL}^{\mathrm{ZS97}}$ & $\mathrm{NIL}^{\mathrm{MY} 46}$ & & & \\
\hline \multirow[t]{5}{*}{ (28 Apr) } & NP & 6.61 & 6.43 & 0.1769 & & & 6.82 & 6.46 & 0.0087 & -0.18 & 3.6 \\
\hline & NGP & 109.4 & 109.4 & 0.9649 & & & 105.2 & 106.7 & 0.3825 & & \\
\hline & TGW & 25.50 & 25.71 & $<0.0001$ & 0.11 & 9.5 & 25.40 & 25.49 & 0.1194 & & \\
\hline & SF & 88.17 & 89.02 & 0.1391 & & & 88.13 & 88.15 & 0.9611 & & \\
\hline & GY & 17.36 & 16.70 & 0.0599 & & & 17.56 & 16.53 & 0.0086 & -0.51 & 3.6 \\
\hline \multirow[t]{5}{*}{ III (20 May) } & NP & 7.16 & 7.15 & 0.9210 & & & 6.65 & 6.71 & 0.6642 & & \\
\hline & NGP & 110.1 & 105.6 & 0.0001 & -2.28 & 7.3 & 115.0 & 108.9 & $<0.0001$ & -3.07 & 9.9 \\
\hline & TGW & 24.74 & 24.56 & 0.0002 & -0.09 & 8.1 & 25.02 & 24.80 & 0.0004 & -0.11 & 8.4 \\
\hline & SF & 83.23 & 83.46 & 0.5555 & & & 83.94 & 84.88 & 0.0418 & 0.47 & 2.0 \\
\hline & GY & 18.55 & 17.72 & 0.0500 & & & 18.32 & 17.57 & 0.0701 & & \\
\hline \multirow[t]{5}{*}{ IV (15 Jun) } & $N P$ & 8.46 & 8.59 & 0.5240 & & & 7.82 & 7.83 & 0.9624 & & \\
\hline & NGP & 101.4 & 98.9 & 0.1892 & & & 106.5 & 105.6 & 0.5625 & & \\
\hline & TGW & 27.04 & 25.06 & $<0.0001$ & -0.99 & 68.3 & 26.73 & 24.36 & $<0.0001$ & -1.19 & 79.6 \\
\hline & SF & 82.11 & 84.20 & 0.0005 & 1.05 & 7.3 & 83.34 & 85.32 & 0.0014 & 0.99 & 5.9 \\
\hline & GY & 20.74 & 18.96 & 0.0006 & -0.89 & 6.6 & 20.57 & 18.37 & $<0.0001$ & -1.10 & 9.8 \\
\hline
\end{tabular}

NP number of panicle per plant, NGP number of grains per panicle, TGW 1000-grain weight (g), SF spikelet fertility rate (\%), GY grain yield per plant (g)

${ }^{\mathrm{a}} \mathrm{NIL}^{\mathrm{ZS} 97}$ and NIL ${ }^{\mathrm{MY} 46}$ are near isogenic lines having homozygous qHd1 alleles from Zhenshan 97 and Milyang 46, respectively

badditive effect of replacing a Zhenshan 97 allele with a Milyang 46 allele

cproportion of phenotypic variance explained by the QTL effect

under SD conditions. As shown in Fig. 2, with the daily mean temperature increase from 22.8 to $24.8^{\circ} \mathrm{C}$, sharp decrease of heading date was observed for both genotypes, i.e., from 114.6 to $76.6 \mathrm{~d}$ for ZS97 homozygotes and from 108.7 to $72.2 \mathrm{~d}$ for MY46 homozygotes. But when further increase to $26.8^{\circ} \mathrm{C}$, stabilization of HD in ZS97 homozygotes were observed with minor decrease of just $1.4 \mathrm{~d}$, in contrary to the great decrease of $7.3 \mathrm{~d}$ in the MY46 homozygotes. Across all the three temperature treatments, highly significant difference in HD $(P<0.0001)$ was found

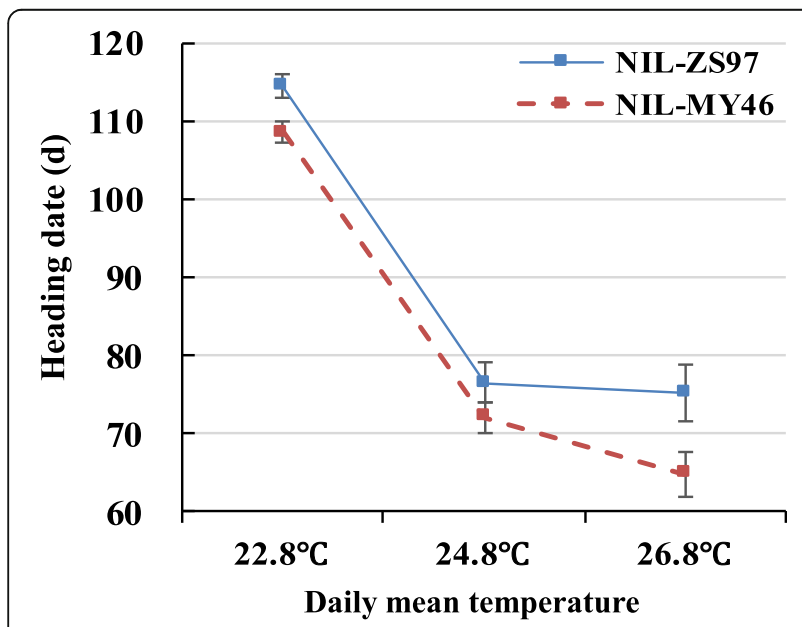

Fig. 2 The temperature-gradient test in phytotron. Heading date of NIL-ZS97 and NIL-MY46 were the averaged data of 16 plants for each genotype. Values are represented as means $\pm \mathrm{SD}$ between the two genotypic groups. These results further indicate the involvement of $q H d 1$ in stabilizing rice heading at high temperatures and possible existence of a thermosensory pathway it mediated. In addition, consistent performance of $q H d 1$ under the SD condition in the phytotron and NLD condition in the paddy field suggests that this thermosensory pathway is independent of day length.

\section{The floral pathway underlying $q H d 1$-mediated thermosensory response}

The target region contains ten annotated genes (http:// rapdb.dna.affrc.go.jp/), including two genes having function related to heading date, i.e., OsMADS51 [31] and OsSPL2 [32]. We sequenced the promoter and entire gene regions of OsMADS51 and OsSPL2 from the two parental lines, ZS97 and MY46. Sequence comparison between ZS97 and MY46 identified no polymorphism in the promoter and exon regions. At the same time, a large insertion of $9.5-\mathrm{kb}$ was detected in the 1 st intron of OsMADS51 in ZS97, as compared to MY46 (Fig. 3 and Additional file 6: Figure S4). This result prompted us to examine whether this large structure variation was a functional polymorphism that has an influence on gene expression. Using qRT-PCR, we tested OsMADS51, and four key heading-date regulators, i.e., florigen genes $H d 3 a$ and RFT1 and their upstream signal integrators Hd1 and Ehd1 [11, 12]. OsSPL2 was also included, because it is located in the $q H d 1$ region and annotated to be functionally related to heading. 


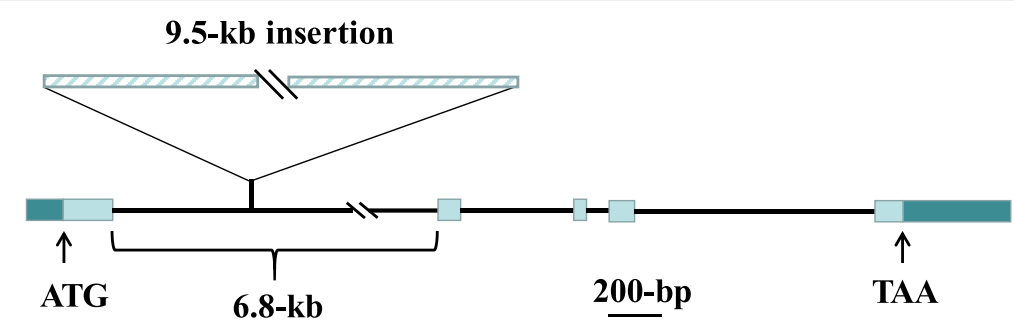

Fig. 3 A large sequence structure variation of $9.5-\mathrm{kb}$ in the 1st intron of OsMADS51. Zhenshan 97 contains a 9.5-kb insertion as compared to Milyang 46

The effect of $q H d 1$ on HD became much larger in Trial IV than the three previous trials (Fig. 1a), indicative of the influence of temperature in heading. Therefore Trial IV was chosen for gene expression analysis. Average HD in this trial was $69.3 \mathrm{~d}$ for ZS97 homozygotes and $61.4 \mathrm{~d}$ for MY46 ones. Gene expression was assessed in 30, 35 and 45-day-old plants grown in the field under NLD conditions, well covering the transition period from vegetative to reproductive phase.

As illustrated in Fig. 4, significant changes in the transcript levels between the two genotypic groups were found for the most potential candidate, OsMADS51, and key heading-date regulators Ehd1, RFT1 and Hd3a. Lower expression was found in NIL-ZS97 than NIL-MY46, which is in accordance with late heading in NIL-ZS97. The largest fold changes were found in the first or second sampling time, including 6.2 fold of OsMADS51 in 30 DAS, 4.6 fold of Ehd1 in 35 DAS, 11.3 fold of RFT1 in 30 DAS, and 3.7 fold of $H d 3 a$ in 30 DAS. These results suggest that the $9.5-\mathrm{kb}$ sequence structure variation in OsMADS51 could underlie a $q H d 1$-mediated heading response to temperature, by regulating the Ehd1-RFT1/ $H d 3 a$ expression.

\section{Distinct expression regulation of $q H d 1$ between high and} low temperatures revealed by RNA-seq

In the phytotron experiment under LD conditions, heading date of the NIL-MY46 and NIL-ZS97 were 48.5 and $64.9 \mathrm{~d}$ under high daily mean temperature of $31.5{ }^{\circ} \mathrm{C}$, and 70.3 and $78.3 \mathrm{~d}$ under low daily mean temperature of $23.5{ }^{\circ} \mathrm{C}$, respectively. The $16.4 \mathrm{~d}$ difference between the two NILs at high temperature was much larger than
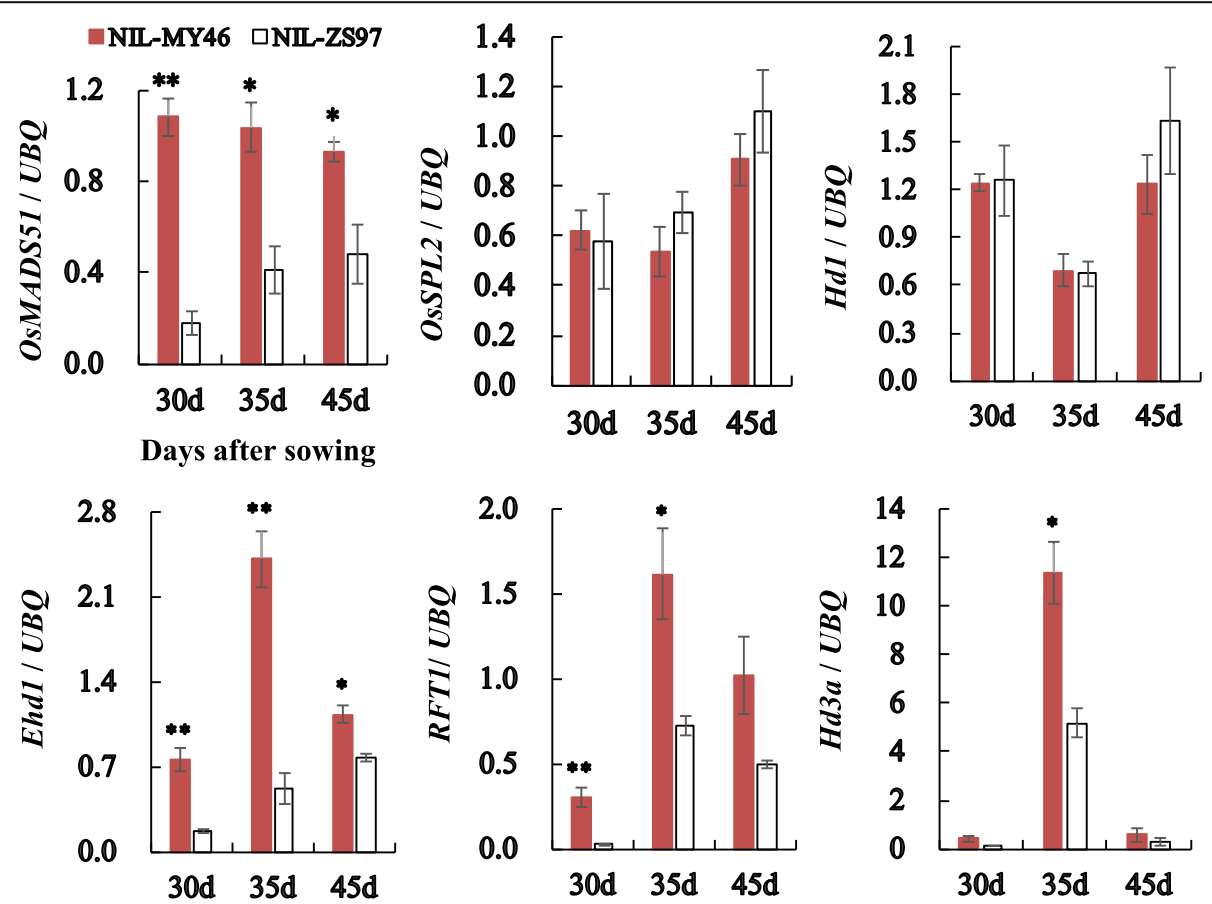

Fig. 4 Comparisons of the gene expression. OsMADS51, OsSPL2, Hd1, Ehd1, RFT1 and Hd3a were tested between ZS97 and MY46 homozygous lines of the CJ1 population by qRT-PCR in 30,35, and 45 days after sowing. Values are represented as means \pm SE, derived from three biological replicates with two technical repetitions each. $U B Q$, ubiqutin used to normalize the values. ${ }^{*} P<0.05$ or ${ }^{* *} P<0.01$, by Student's t-test 
the value of $8.0 \mathrm{~d}$ at low temperature, showing the response of $q H d 1$ to temperature variation. It is also noted that the underlying gene OsMADS51 encoded an MADS-box transcription factor, regulating the expression of genes that are related to metabolism and signaling in response to environmental variations [33, 34]. Therefore, RNA-seq was performed to identify downstream genes involved in $q H d 1$-mediated thermosensory network.

Differentially expressed genes (DEGs) between NIL-ZS97 and NIL-MY46 were identified (Additional file 7: Table S3), showing large differences between the two treatments (Fig. 5). Ninety-nine DEGs were detected at high temperature, much fewer than 287 DEGs found at low temperature (Fig. 5a). As compared to NIL-MY46, the numbers of DEGs up- and down-regulated in NIL-ZS97 were 80 and 19 at high temperature, but 68 and 219 at low temperature (Fig. 5b). In addition, for the category of up-regulated genes at
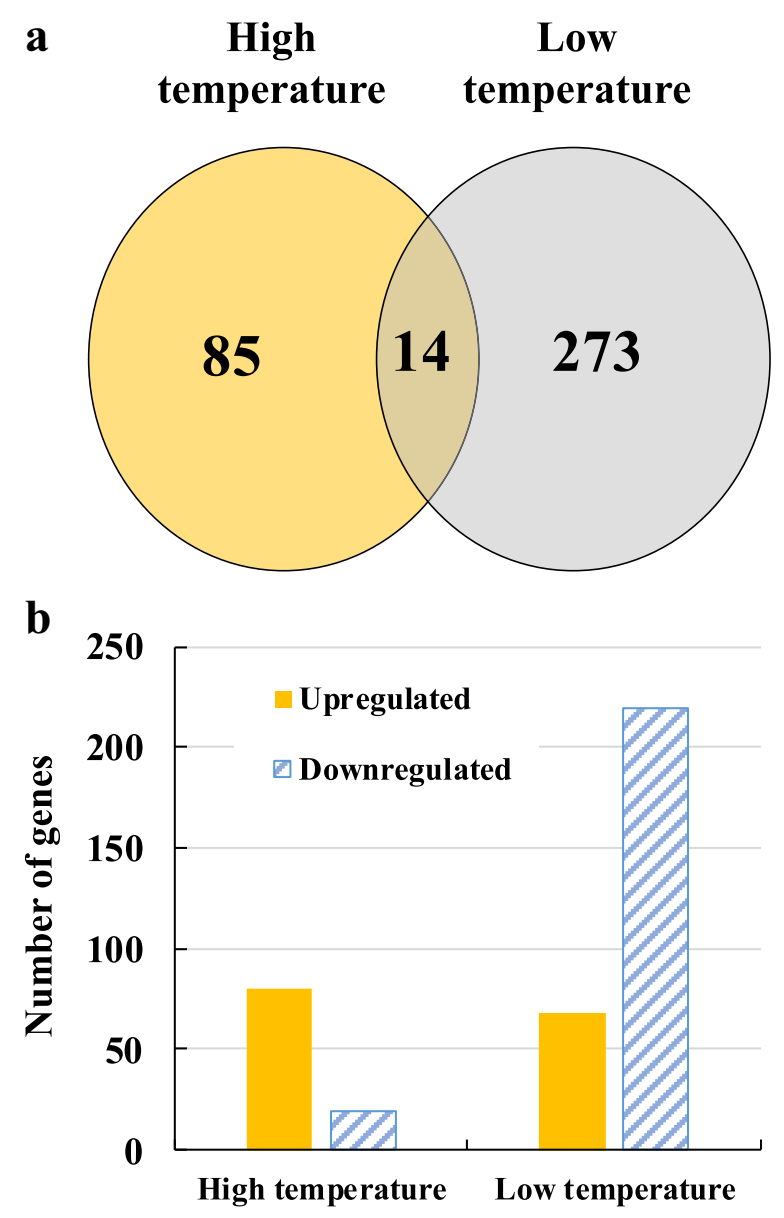

Fig. 5 Overview of differentially expressed genes identified by RNA-seq. a Venn diagram illustrates unique and common genes differentially regulated in the high- and low-temperature treatment. b Histogram shows the number of genes up-regulated and downregulated in the high- and low-temperature treatments of NIL-ZS97 as compared to NIL-MY46 high-temperature treatment, seven of the top ten genes showing the largest expression change were found to participate in stress responses, including four genes related to cold conditions (Additional file 8: Table S4). For the remaining three category of DEGs, few of the top ten genes responded to adverse temperature stresses. These results implied that the expression-regulation pattern of $q H d 1$ may be differentiated in response to temperature variation, and further confirm the involvement of $q H d 1$ in thermosensory pathway.

Of the DEGs identified, only 14 were commonly found in the two treatments. While OsMADS51, Ehd1 and RFT1 were detected as common DEGs, no other heading-date genes exhibited significant differential expression. Considering that high homology and close linkage between RFT1 and $H d 3 a$ may prevent the separate detection of them, $H d 3 a$ was subject to qRT-PCR analysis using RNA samples applied for RNA-seq, together with OsMADS51, Ehd1 and RFT1. All the four genes showed significant expression differences as expected (Additional file 9: Figure S5), suggesting a new pathway OsMADS51-Ehd1-RFT1/Hd3a for the regulation of flowering in response to temperature.

\section{Differentiation of $q \mathrm{Hd} 1$ between early-season and middle/ late-season indica rice varieties}

As described above, the $q H d 1$ allele derived from the early-season indica rice variety ZS97 conferred tolerance to high temperature which is a common environmental stress encountered during the heading and grain-filling period of early-season rice cropping. We therefore decided to test whether the beneficial allele with the 9.5-kb insertion has been commonly utilized in rice production. One-hundred and nine indica rice varieties widely used in China were collected and analyzed (Additional file 3: Table S1).

Eleven pairs of PCR primers were used for genotyping of $q H d 1$. Ten pairs extending from $1 \mathrm{~F} / 1 \mathrm{R}$ to $10 \mathrm{~F} / 10 \mathrm{R}$ jointly spanned the entire 9.5-kb insertion of the ZS97 allele (Fig. 3 and Additional file 6: Figure S4). Meanwhile, primer pair $1 \mathrm{~F} / 10 \mathrm{R}$ amplified a $1.0-\mathrm{kb}$ fragment from MY46. Among the 13 early-season indica rice varieties that had large planting areas for a long period before 1985, all except one carried the 9.5-kb insertion. Among the 82 major indica rice varieties used during 1990-2014, the 9.5-kb insertion was identified in 43 of the 48 early-season varieties, but only detected in three of the 24 middle-season and two of the ten late-season varieties. For the 14 early-season indica rice varieties released after 2000, including the backbone parents Jiayu 253 and Zhongxuan 181, and 12 descendants of them, the $9.5-\mathrm{kb}$ insertion was found without exception. These findings indicate that $q H d 1 / O s M A D S 51$ could have undergone intensive artificial selection and play a significant role in the adaptation of early-season indica rice to high temperature at the heading and grain-filling stages. 


\section{Discussion}

High temperature commonly has negative impacts on rice development and causes yield losses. Rice heading is generally accelerated when temperature increased. The shortened vegetative-growth duration accompanied by limited source build-up is often a severe constraint for attaining a high yield [35]. During the reproductive growth and grain-filling stages, elevated temperature could result in reduced starch accumulation in developing grain because of the declined starch biosynthesis and earlier cessation of assimilate translocation [36]. Identification of genes associated with tolerance to heading date acceleration is of great importance for rice breeding. In the present study, we identified the thermosensory features of $q H d 1$, a minor QTL for heading date that was preciously fine-mapped [19]. Results showed that the allele derived from the early-season indica rice variety ZS97 conferred stable heading date with higher grain weight and grain yield under high temperatures. It was also found that this allele is predominantly shared by early-season indica rice varieties, but few middle- and late-season rice varieties carry this allele. Considering the frequent occurrence of high temperature stress in both the early- and middle-season rice cropping especially in recent years, introgression of the beneficial allele at $q H d 1$ from early-season rice to middle-season rice would be greatly helpful for overcoming yield losses due to heat stresses.

Another finding of this study is the OsMADS51-Ehd1-RFT1/Hd3a pathway underlying the qHd1-mediated floral response to temperature. The Ehd1-RFT1/Hd3a pathway downstream of OsMADS51 also functioned as integrators of drought signals [37]. The convergence of heat and drought signal on the heading-date pathway implied the regulatory connection between stress and flowering. As it is well-known, plants generally perceive and integrate various environmental signals to determine the proper timing of phase transition for maximizing reproductive success, either rapidly induce flowering before the stress becomes a great detrimental or temporarily inhibit heading until the stress is over [38]. Consistent with this view, Grain number, plant height and heading date 7 (Ghd7), the major floral repressors upstream of Ehd1 and the two florigen genes in rice [39], was reported to respond to multiple abiotic stress, including drought, abscisic acid, jasmonic acid, high temperature and low temperature [40]. Likewise, in our results of transcriptome profiling, the OsMADS51/qHd1-mediated expression change was observed for a group of genes that were involved in multiple abiotic and biotic stress-response pathways such as cold, heat, drought, salinity, disease and insect (Additional file 8: Table S4). These results suggest that the heading-date genes/pathway might play a key role in integrating the floral transition and stress response, which would provide new leads for exploring the intricate genetic networks.
Day length and temperature are two key environmental factors associated with seasonal changes and strongly affect the crop duration and grain yield of rice. Recent studies have revealed that heading-date genes could possessed pleiotropism for yield traits, and their natural variations have been used in rice breeding mainly with the late-maturing allele enhancing grain yield, such as Ghd7 [39], Days to heading 8/Grain number, plant height and heading date 8 [41, 42], Hd1 [43], Grain number, plant height and heading date 7.1 /Days to heading 7 [44, 45], and RFT1 [46]. These reports focus on the pleiotropism for yield traits in response to photoperiodic changes, and none of them work on the response to temperature variation. In the multiple sowing-date experiment of this study, we noted the pleiotropism of OsMADS51/qHd1 varied greatly in response to temperature change (Table 3 ). Variation in grain yield might be derived from both NGP and TGW (Trial III) or mainly from TGW (Trial IV). The magnitude of genotypic effects on yield traits also varied greatly, showing an association of stronger effects with increased daily-mean temperature (Additional file 5: Figure S3). This finding was consistent with the reports that a slight temperature change of $1-2{ }^{\circ} \mathrm{C}$ would have dramatic effects on grain yield and its components [2, 3, 47].

OsMADS51, the gene underlying $q H d 1$, was previously isolated by T-DNA insertion and reported to be photoperiod sensitive, influencing heading date under SD conditions only [31]. However, this gene also showed contribution to heading-date variation under LD conditions in this study. These suggest that OsMADS51 could affect heading under both under the SD and LD conditions, but the function may change depending on the genetic backgrounds. For the temperature responsive function of OsMADS51, no similar result has been reported for heading date genes in rice. Nevertheless, it is noted that OsMADS51 encodes a MADS-domain transcription factor, and several key components such as SHORT VEGETATIVE PHASE [48], FLOWERING LOCUS M [49], FLOWERING LOCUS $C$ and MADS AFFECTING FLOWERING 2, 3, 4 and 5 [50] in the Arabidopsis flowering-regulatory thermosensory pathway also belong to the MADS-box transcription factor family. This is in support of the inference that OsMADS51 mediates the floral response to temperature variation in rice.

\section{Conclusions}

Our work identified the genetic contribution of $q H d 1$ to temperature responses in rice, showing that this QTL regulates heading date in association with high-temperature tolerance at the heading and grain-filling stages. The underlying floral pathway OsMADS51-Ehd1-Hd3a/RFT1 was found, in which OsMADS51 is the gene for QTL 
qHd1. The beneficial allele of OsMADS51 has become predominant in early-season indica rice varieties but remains rare in the middle- and late-season ones. This indicates the great importance of $q H d 1$ for the adaptation of early-season indica rice to high temperature during the yield-forming process. The information provides new leads for a molecular understanding of how temperature influences rice growth and then affects rice production.

\section{Additional files}

Additional file 1: Figure S1. Frequency distribution of heading date in the two NIL populations tested in Lingshui in Dec 2013-Apr 2014. NILZS97 and NIL-MY46 are near isogenic lines with Zhenshan 97 and Milyang 46 homozygous genotypes at qHd1, respectively. (PPT $119 \mathrm{~kb}$ )

Additional file 2: Figure S2. Development of two sets of near isogenic lines segregated for $\mathrm{qHd}$ land genotypic compositions of the two populations in the target region. (PPT $118 \mathrm{~kb}$ )

Additional file 3: Table S1. Three collections of Chinese indica rice varieties tested in this study. (XLSX $17 \mathrm{~kb}$ )

Additional file 4: Table S2. Sequences of the primers used for quantitative real-time PCR. (DOCX $13 \mathrm{~kb}$ )

Additional file 5: Figure S3. Daily mean temperature and day length variation observed in the multiple-sowing experiment conducted in Hangzhou in 2015. The data of daily mean temperature were derived from the weather station located near the experimental field, while day length data were from www.timeanddate.com. I, II, III, IV and V refer to different trials with sowing date of 28 Apr, 8 May, 20 May, 15 Jun and 9 Jul, respectively. Gray columns represent the duration from sowing to heading in each trial, with the corresponding averaged temperature. (PPT $132 \mathrm{~kb}$ )

Additional file 6: Figure S4. Genomic sequence of OSMADS51 in Zhenshan 97 , showing the $9.5-\mathrm{kb}$ insertion as compared to Milyang 46. The sequence of $3^{\prime}$ and $5^{\prime}-$ UTR is shaded in green, exons in yellow, and the $9.5-\mathrm{kb}$ insertion in grey. Ten pairs of primers used for sequencing and genotyping of the $9.5-\mathrm{kb}$ insertion is indicated by colored and underlined letters, with forward primers in red and reverse primers in golden, e.g., 1F/1R - 10F/10R. (PDF $449 \mathrm{~kb})$

Additional file 7: Table S3. Differentially expressed genes detected by RNA-seq in high- and low-temperature treatment, classified by regulation direction and ranked by log2FC. (XLSX $118 \mathrm{~kb})$

Additional file 8: Table S4. Differentially expressed genes responding to adverse stress. (XLSX $16 \mathrm{~kb}$ )

Additional file 9: Figure S5. Validation of RNA-seq profile in the hightemperature (a) and low-temperature (b) treatment by expression analysis of OsMADS51, Ehd1, RFT and Hd3a using qRT-PCR. Values are represented as means $\pm \mathrm{SE}$, derived from two biological replicates with two technical repetitions each. $U B Q$, ubiqutin used to normalize the values. (PPT $116 \mathrm{~kb}$ )

\section{Acknowledgements}

We thank the National Medium Rice Genebank at the China National Rice Research Institute for providing rice accessions.

\section{Funding}

This work was supported by grants from the National Natural Science Foundation of China $(31701398,31371605)$ and the Chinese 863 Program (2014AA10A604-15)

\section{Availability of data and materials}

RNA-seq data are available from the Short Read Archive under accession number SRP115036. Any other datasets used and/or analyzed during the current study available from the corresponding author on reasonable request.

\section{Authors' contributions}

JYZ designed the experiments. JYZ and LYM selected the rice materials. JYC performed most of the experiments. HWZ, HLZ and LYM performed some of the phenotyping in the field. JZY performed some of the phenotyping in the phytotron. JYC and JYZ analyzed the data and wrote the paper. All authors have read and approved this manuscript.

Ethics approval and consent to participate

Not applicable

\section{Competing interests}

The authors declare that they have no competing interests.

\section{Publisher's Note}

Springer Nature remains neutral with regard to jurisdictional claims in published maps and institutional affiliations.

Received: 9 February 2018 Accepted: 24 May 2018

Published online: 07 June 2018

\section{References}

1. Zhang Z, Wang $P$, Chen $Y$, Song $X$, Wei $X$, Shi P. Global warming over 1960-2009 did increase heat stress and reduce cold stress in the major rice-planting areas across China. Eur J Agron. 2014;59:49-56.

2. Shah F, Nie L, Cui K, Shah T, Wu W, Chen C, et al. Rice grain yield and component responses to near $2^{\circ} \mathrm{C}$ of warming. Field Crops Res. 2014; 157:98-110.

3. Peng S, Huang J, Sheehy J, Laza R, Visperas R, Zhong X, et al. Rice yield decline with higher night temperature from global warming. Proc Natl Acad Sci U S A. 2004;101:9971-5.

4. Yoshida S. Fundamentals of rice crop science. Los Baños: IRRI; 1981.

5. Chang T, Li C, Vergara B. Component analysis of duration from seeding to heading in rice by the basic vegetative phase and the photoperiodsensitive phase. Euphytica. 1969;18:79-91.

6. Hsieh S, Chang T. Radiation induced variations in photoperiod-sensitivity, thermo-sensitivity and the number of days to heading in rice. Euphytica. 1975;24:487-96.

7. Wei X, Xu J, Jiang L, Wang H, Zhou Z, Zhai H, Wan J. Genetic analysis for the diversity of heading date of cultivated rice in China. Acta Agron Sin. 2012;38:10-2. (in Chinese with English abstract)

8. Morita S, Yonemaru J, Takanashi J. Grain growth and endosperm cell size under high night temperatures in rice (Oryza sativa L.). Ann Bot. 2005;5:695-701.

9. Cao Y, Duan H, Yang L, Wang Z, Zhuo S, Yang J. Effective heat stress during meiosis on grain yield of rice cultivars differing in heat tolerance and its physiological mechanism. Acta Agron Sin. 2008;34:2134-42. (in Chinese with English abstract)

10. Jagadish S, Craufurd P, Wheeler T. Phenotyping parents of mapping populations of rice (Oryza sativa L.) for heat tolerance during anthesis. Crop Sci. 2008;48:1140-6.

11. Song Y, Shim J, Kinmonth-Schultz H, Imaizumi T. Photoperiodic flowering: time measurement mechanisms in leaves. Annu Rev Plant Biol. 2015;66:441-64.

12. Hori K, Matsubara K, Yano M. Genetic control of flowering time in rice: integration of Mendelian genetics and genomics. Theor Appl Genet. 2016; 129:2241-52.

13. Koini M, Alvey L, Allen T, Tilley C, Haeberd N, Whitelam G, et al. High temperature-mediated adaptations in plant architecture require the bHLH transcription factor PIF4. Curr Biol. 2009;19:408-13.

14. Strasser B, Alvarez M, Califano A, Cerdan P. A complementary role for ELF3 and TFL1 in the regulation of flowering time by ambient temperature. Plant J. 2009;58:629-40

15. Ma D, Li X, Guo Y, Chu J, Fang S, Yan C, et al. Cryptochrome 1 interacts with PIF4 to regulate high temperature-mediated hypocotyl elongation in response to blue light. Proc Natl Acad Sci U S A. 2016;113:224-9.

16. Sanchez-Bermejo E, Zhu W, Tasset C, Eimer H, Sureshkumar S, Singh R, et al. Genetic architecture of natural variation in thermal responses of Arabidopsis. Plant Physiol. 2015;169:647-59.

17. Jung J, Domijan M, Klose C, Biswas S, Ezer D, Gao M, et al. Phytochromes function as thermosensors in Arabidopsis. Science. 2016;354:886-9. 
18. Legris M, Klose C, Burgie ES, Costigliolo C, Neme M, Hiltbrunner A, et al. Phytochrome B integrates light and temperature signals in Arabidopsis. Science. 2016;354:897-900.

19. Chen J, Guo L, Ma H, Chen Y, Zhang H, Ying J, Zhuang J. Fine mapping of qHd1, a minor heading date QTL with pleiotropism for yield traits in rice (Oryza sativa L.). Theor Appl Genet. 2014;127:2515-24.

20. China Technique and Extension Service Centre. Extension Statue of Major Varieties of Crops in China. Beijing: Ministry of Agriculture; 1981-2014. (in Chinese).

21. Wei X, Tang S. Inbred rice varieties in China. Zhejiang: Science and Technology Press; 2011. (in Chinese)

22. Takimoto A, Ikeda K. Effect of twilight on photoperiodic induction in some short day plants. Plant Cell Physiol. 1961;2:213-29.

23. Nishida $H$, Inoue $H$, Okumoto $Y$, Tanisaka $T$. A novel gene ef1- $h$ conferring an extremely long basic vegetative growth period in rice. Crop Sci. 2002;42:348-54.

24. Itoh $\mathrm{H}$, Nonoue $\mathrm{Y}$, Yano M, Izawa $\mathrm{T}$. A pair of floral regulators sets critical day length for Hd3a florigen expression in rice. Nat Genet. 2010;42:635-8.

25. Zhang H-W, Fan Y-Y, Zhu Y-J, Chen J-Y, Yu S-B, Zhuang J-Y. Dissection of the aTGW1.1 region into two tightly-linked minor QTLs having stable effects for grain weight in rice. BMC Genet. 2017;17:98.

26. Livak K, Schmittgen T. Analysis of relative gene expression data using realtime quantitative PCR and the $2^{-\Delta \Delta C T}$ method. Methods. 2001;25:402-8.

27. Dai W, Zhang K, Wu J, Wang L, Duan B, Zheng K, et al. Validating a segment on the short arm of chromosome 6 responsible for genetic variation in the hull silicon content and yield traits of rice. Euphytica. 2008;160:317-24.

28. Vergara B, Chang T. The flowering response of the rice plant to photoperiod. $4^{\text {th }}$ edn. LosBaños, Philippines: IRRI; 1985.

29. Yin X, Kropff M, Goudriaan J. Changes in temperature sensitivity of development from sowing to flowering in rice. Crop Sci. 1997;37:1787-94.

30. Liu T, Liu H, Zhang H, Xing Y. Validation and characterization of Ghd7.1, a major quantitative trait locus with pleiotropic effects on spikelets per panicle, plant height, and heading date in rice (Oryza sativa L.). J Integr Plant Biol. 2013;55:917-27.

31. Kim S, Lee $\mathrm{S}, \mathrm{Kim} H$, Nam H, An G. OsMADS51 is a short-day flowering promoter that functions upstream of Ehd1, OsMADS14 and Hd3a. Plant Physiol. 2007;145:1484-94.

32. Huijser $P$, Schmid M. The control of developmental phase transitions in plants. Development. 2011;138:4117-29.

33. Messenguy F, Dubois E. Role of MADS box proteins and their cofactors in combinatorial control of gene expression and cell development. Gene. 2003;316:1-21

34. Arora R, Agarwal P, Ray S, Singh AK, Singh VP, Tyagi AK, Kapoor S. MADSbox gene family in rice: genome-wide identification, organization and expression profiling during reproductive development and stress. BMC Genomics. 2007:8:242.

35. Hu Z, Liu Y, Huang L, Peng S, Nie L, Cui K, Huang J, Wang F. Premature heading and yield losses caused by prolonged seedling age in double cropping rice. Field Crop Res. 2015;183:147-55.

36. Farooq $\mathrm{M}$, Bramley $\mathrm{H}$, Palta J, Siddique K. Heat stress in wheat during reproductive and grain filling phases. Plant Sci. 2011;30:491-507.

37. Galbiati F, Chiozzotto R, Locatelli F, Spada A, Genga A, Fornara F. Hd3a, RFT1 and Ehd 1 integrate photoperiodic and drought stress signals to delay the floral transition in rice. Plant Cell Environ. 2016;39:1982-93.

38. Andrés F, Coupland $\mathrm{G}$. The genetic basis of flowering responses to seasonal cues. Nat Rev Genet. 2012;13:627-39.

39. Xue $W$, Xing $Y$, Weng $X$, Zhao $Y$, Tang $W$, Wang $L$, et al. Natural variation in Ghd7 is an important regulator of heading date and yield potential in rice. Nat Genet. 2008;40:761-7.

40. Weng X, Wang L, Wang J, Hu Y, Du H, Xu C, et al. Grain number, plant height, and heading date 7 is a central regulator of growth, development, and stress response. Plant Physiol. 2014;164(2):735-47.

41. Wei X, Xu J, Guo H, Jiang L, Chen S, Yu C, et al. DTH8 suppresses flowering in rice, influencing plant height and yield potential simultaneously. Plant Physiol. 2010. 2010;153:1747-58.

42. Yan W, Wang P, Chen H, Zhou H, Li Q, Wang C, et al. A major QTL, Ghd8, plays pleiotropic roles in regulating grain productivity, plant height, and heading date in rice. Mol Plant. 2011;4:319-30.

43. Zhang Z, Wang K, Guo L, Zhu Y, Fan Y, Cheng S, Zhuang J. Pleiotropism of the photoperiod-insensitive allele of $\mathrm{Hd} 1$ on heading date, plant height and yield traits in rice. PLoS One. 2012;7:e52538.
44. Yan W, Liu H, Zhou X, Li Q, Zhang J, Lu L, et al. Natural variation in Ghd7.1 plays an important role in grain yield and adaptation in rice. Cell Res. 2013; 23:969-71.

45. Gao H, Jin M, Zheng X, Chen J, Yuan D, Xin Y, et al. Days to heading 7, a major quantitative locus determining photoperiod sensitivity and regional adaptation in rice. Proc Natl Acad Sci U S A. 2014;111:16337-42.

46. Zhu Y, Fan Y, Wang K, Huang D, Liu W, Ying J, Zhuang J. Rice Flowering Locus $T 1$ plays an important role in heading date influencing yield traits in rice. Sci Rep. 2017;7:4918.

47. Dong W, Chen J, Zhang B, Tian Y, Zhang W. Responses of biomass growth and grain yield of midseason rice to the anticipated warming FATI facility in East China. Field Crop Res. 2011;123:259-65.

48. Lee J, Yoo S, Park S, Hwang I, Lee J, et al. Role of SVP in the control of flowering time by ambient temperature in Arabidopsis. Genes Dev. 2007;21:397-402.

49. Balasubramanian S, Sureshkumar S, Lempe J, Weigel D. Potent induction of Arabidopsis thaliana flowering by elevated growth temperature. PLoS Genet. 2006;2:e106.

50. Gu X, Le C, Wang Y, Li Z, Jiang D, Wang Y, et al. Arabidopsis FLC clade members form flowering-repressor complexes coordinating responses to endogenous and environmental cues. Nat Commun. 2013;4:1947.

\section{Ready to submit your research? Choose BMC and benefit from:}

- fast, convenient online submission

- thorough peer review by experienced researchers in your field

- rapid publication on acceptance

- support for research data, including large and complex data types

- gold Open Access which fosters wider collaboration and increased citations

- maximum visibility for your research: over $100 \mathrm{M}$ website views per year

At BMC, research is always in progress.

Learn more biomedcentral.com/submissions 